\title{
Combined Neural Network and PD Adaptive Tracking Controller for Ship Steering System
}

\author{
Abdul-Basset Al- Hussein \\ Electrical Engineering Department \\ College of Engineering \\ Basrah University \\ Basrah, Iraq.
}

Abdulbasset.alhussein@gmail.com

\begin{abstract}
In this paper, a combined RBF neural network sliding mode control and PD adaptive tracking controller is proposed for controlling the directional heading course of a ship. Due to the high nonlinearity and uncertainty of the ship dynamics as well as the effect of wave disturbances a performance evaluation and ship controller design is stay difficult task. The Neural network used for adaptively learn the uncertain dynamics bounds of the ship and their output used as part of the control law moreover the PD term is used to reduce the effect of the approximation error inherited in the RBF networks. The stability of the system with the combined control law guaranteed through Lyapunov analysis. Numeric simulation results confirm the proposed controller provide good system stability and convergence.
\end{abstract}

Index Terms- NEURAL NETWORK, ADAPTIVE CONTROL, SLIDING MODE CONTROL, MODEL REFERENCE PD CONTROLLER.

\section{INTRODUCTION}

An autopilot is a ship steering controller which is an important equipment for ship maneuvering, and automatically manipulates the rudder angle to reduce the error between the reference course or heading and actual course. The autopilot performance directly effect safety and fuel want consumption. Ship autopilot designed by conventional controller must be adjusted in accordance with the variation of ship dynamic and environmental disturbances where ship dynamic are greatly influences by ships speed, type ballast condition, so, the ship model has a high degree of nonlinearity and uncertainty, and this is beside the environmental effect namely the wind and currents $[1,2]$. To address the major difficulties of the ship nonlinearity and uncertainty, several techniques have been developed, J. Van Ameronge [3] proposed adaptive model reference approach. Lavudal and Fossen [4] suggested robust adaptive autopilot with waves filter and integral action. Other researches, a class of recursive algorithms such as backstepping and filtered backstepping have been applied [5]. In [2] an adaptive neural network control for ship steering system is proposed and backstepping techniques has been used. A fuzzy variable structure controller is designed based on exponential reaching law presented in [6]. While a model reference based neural network is used in [7]. In [8] J. R. Layne and K. Passino had proposed a fuzzy reference model learning control for steering cargo ship system.

In this Paper, a combined neural network and PD controller is proposed. The neural network is used to adaptively learning ship uncertainly bound, and the output is used as a parameter in the control law. To reduce the effect of the unknown dynamics of the ship; a PD term has been added to the control law. The PD term will compensate the model uncertainty outside the neural network state region, this provide global stability, moreover, the PD term handles the inherent network approximation error and this will improve the tracking. The asymptotical stability of the system including both terms of the control law the sliding and the PD terms has been approved using Lyapunov theorem beside the derivation of the learning algorithm of the neural networks. 


\section{The ShIP Mathematical Model}

Ship maneuverability means the ability of ship to maintain or change its course or heading angle [9]. Ship plane motion is defined in the relative coordinates system which is fixed to the ship as shown in Fig. 1.

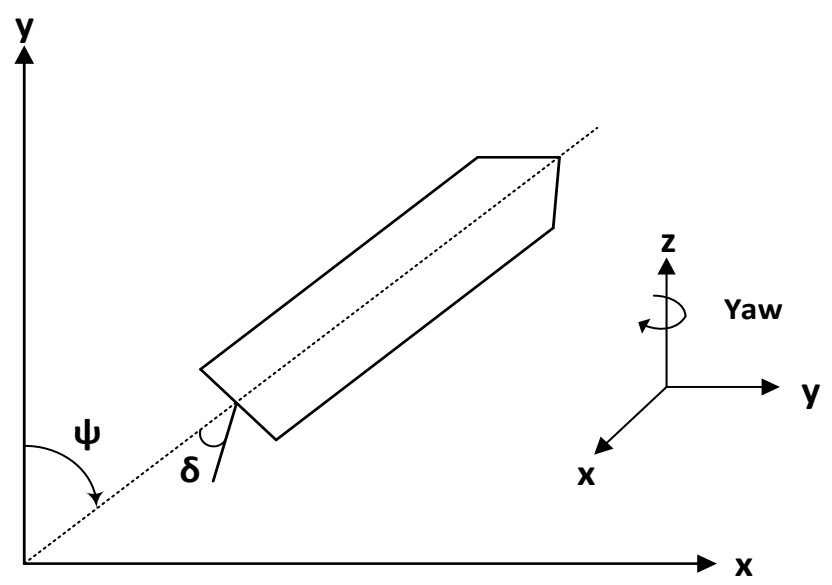

Fig. 1 Ship Coordinates System

Considering the nonlinearity form in the ship dynamics, the mathematical model can be written in the following general form:

$\ddot{\psi}(t)+d_{3} \dot{\psi}^{3}(t)+d_{2} \dot{\psi}^{2}(t)+d_{1} \dot{\psi}(t)+d_{0}=$ $k \delta(t)$

where $\psi$ denotes ship course or heading angle, $\delta$ is the rudder angle. Eq. (1) can be written in a general state model as below:

$\dot{x}=A x+B \delta(t)+F$

where

$\boldsymbol{x}=\left[\psi(t), \dot{\psi}(t), \ldots \psi^{(n-1)}(t)\right]^{T}$

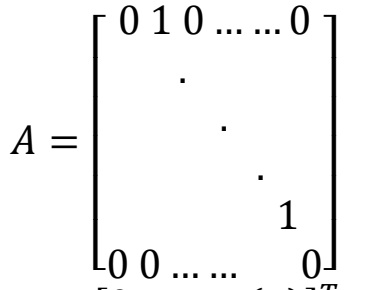

$B=[0, \ldots, K(\boldsymbol{x})]^{T}$

$F=[0, \ldots,-d(\boldsymbol{x})]^{T}$.

where $\boldsymbol{x}, B$ and $F \in \mathfrak{R}^{n}$ and $A \in \mathfrak{R}^{n * n}$ while $\psi^{(i)}$ is the ith time derivative of $\psi(t)$ and $d\left(\psi(t), \dot{\psi}(t), \ldots \psi^{(n-1)}(t)\right)$ and

$K\left(\psi(t), \dot{\psi}(t), \ldots \psi^{(n-1)}(t)\right)$ are the unknown nonlinear functions, with the following properties:

$$
\begin{aligned}
d(\psi(t), \dot{\psi}(t), & \left.\ldots \psi^{(n-1)}(t)\right) \\
& <d_{u}\left(\psi(t), \dot{\psi}(t), \ldots \psi^{(n-1)}(t)\right)
\end{aligned}
$$

Where $d_{u}\left(\psi(t), \dot{\psi}(t), \ldots \psi^{(n-1)}(t)\right)$ is a positive function. While the sign of the control gain $K\left(\psi(t), \dot{\psi}(t), \ldots \psi^{(n-1)}(t)\right)$ is know $(K()>0$. and it is lower bounded:

$$
\begin{aligned}
K(\psi(t), \dot{\psi}(t) & \left., \ldots \psi^{(n-1)}(t)\right) \\
& >K_{l}\left(\psi(t), \dot{\psi}(t), \ldots \psi^{(n-1)}(t)\right)
\end{aligned}
$$

\section{SHIP STEERING CONTROL}

An appropriate desired reference model is proposed by Van Amerongen [3]:

$\ddot{\psi_{m}}+a_{m} \dot{\psi_{m}}+b_{m} \psi_{m}=k_{m} \psi_{r}$

Where $\psi_{m}$ represents the desired system performance of the ship heading. The dynamic of the desired reference model must be matched to the dynamics of the ship in spite of the demanded magnitude of the change of reference course angle.

Eq. (5) can be expressed in the state model as:

$\dot{\boldsymbol{x}}_{m}=A_{m} \boldsymbol{x}_{m}+B_{m} \psi_{r}(t)$

where

$\boldsymbol{x}_{m}=\left[\psi_{m}(t), \dot{\psi_{m}}(t), \ldots \psi_{m}^{(n-1)}(t)\right]^{T}$

with $\boldsymbol{x}_{m} \in \mathfrak{R}^{n}$ is the reference model state and $A_{m} \in \mathfrak{R}^{n * n}$ and $B_{m} \in \mathfrak{R}^{n}$ are the reference model matrices.

The objective of the controller is to obtain a control law and updating law of the controller parameters, such that the whole system responds dynamically as the required reference model.

It is supposed that the ship course tracking error is

$\boldsymbol{e}(t)=\boldsymbol{x}-\boldsymbol{x}_{m}$ 
Then the closed loop system equation in term of the error is given by:

$\dot{\boldsymbol{e}}=A \boldsymbol{e}+\left(A-A_{m}\right) \boldsymbol{x}_{m}+F-B_{m} \psi_{r}+B \delta(t)$

Moreover a sliding mode switching variable defined below:

$s=\mathrm{C} \boldsymbol{e}$

where $\mathrm{C}=\left[c_{1}, c_{2}, \ldots \ldots c_{n}\right]$, with $\mathrm{C} \in \mathfrak{R}^{n}$ is a design parameters and selected to adjust the switching polynomial's zeros in the left half plane. Usually, $\mathrm{C} e$ is called the sliding mode control variable.

Let us consider the ship dynamics upper and lower bounds $d_{u}(\boldsymbol{x})$ and $K_{l}(\boldsymbol{x})$ such that:

$\Phi_{1}=K_{l}^{-1}(\boldsymbol{x})$

$\Phi_{2}=d_{u}(\boldsymbol{x})$

Then will be replaced by the RBF neural networks.

$\widehat{\Phi}_{1}\left(x \mid \widehat{\Theta}_{1}\right)=\widehat{\Theta}_{1}^{T} \zeta_{1}(x)$

$\widehat{\Phi}_{2}\left(x \mid \widehat{\Theta}_{2}\right)=\widehat{\Theta}_{2}^{T} \zeta_{2}(x)$

With $\widehat{\Theta}_{1} \in \mathfrak{R}^{N_{1}}$ and $\widehat{\Theta}_{2} \in \mathfrak{R}^{N_{2}}$ are the interconnection weights vectors, where $N_{1}$ and $N_{2}$ are number of the neurons in the output layer of the first and second neural networks respectively. $\zeta_{1}(\boldsymbol{x}) \in \mathfrak{R}^{N_{1}}$ and $\zeta_{2}(\boldsymbol{x}) \in$ $\mathfrak{R}^{N_{2}}$ are the Gaussian type basis function defined by:

$\zeta_{j}(\boldsymbol{x})=\exp \left(\frac{\left\|x-C_{j}\right\|^{2}}{2 \sigma_{j}^{2}}\right) \quad j=1,2$

where $C_{j}$ is the mean vector and $\sigma_{j}$ is the standard deviation vector of the Gaussian basis functions. The theoretical analysis of the Gaussian networks usually assumes that the basis function are evenly distributed on an n-dimensional lattice with the mean of a basis function located at every point on the lattice. In practical application there are many methods can be used to select the appropriate parameter of the basis function, such as the PSO, genetic algorithms and an unsupervised competitive clustering algorithm which used for on-line adjustment of the mean and deviation of the Gaussian network.

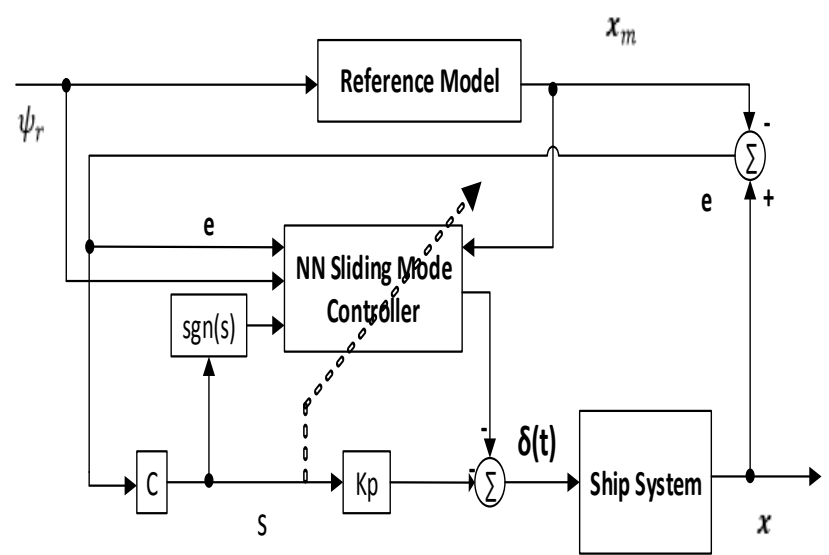

Fig. 2 The Proposed NNSMC sliding mode and PD controller

The proposed control law is consisting of two parts, the first part is depending on a variable gain sliding mode control algorithm using neural network and reference model, while second term uses the filtered error as a PD controller which efficiently can reduce the effect of the approximation error in the neural network as follow:

$$
\begin{aligned}
\delta(t)=-K_{p} s & -\frac{\operatorname{sgn}(s)}{c_{n}}\left(\widehat{\Phi}_{1}\left(\boldsymbol{x} \mid \widehat{\Theta}_{1}\right)\right)^{2}[|C A \boldsymbol{e}| \\
& \left.+\left|C\left(A-A_{m}\right) \boldsymbol{x}_{m}\right|+\left|C B_{m} \psi_{r}\right|\right] \\
& -\operatorname{sgn}(s)\left(\widehat{\Phi}_{1}\left(\boldsymbol{x} \mid \widehat{\Theta}_{1}\right) \widehat{\Phi}_{2}\left(\boldsymbol{x} \mid \widehat{\Theta}_{2}\right)\right)
\end{aligned}
$$

where $K_{p}$ is a design parameter. The whole system is shown in Fig. 2

Theorem: Considering the error dynamics (8) for the ship steering system and the desired control input (15), the ship steady steering system asymptotically tracks with the desired signal so that the system error converge to zero [11. 12].

Proof: A Lyapunov function is defined as follows:

$L(s, \widetilde{\Theta})=\frac{1}{2} s^{2}+\frac{1}{2} \Gamma_{1}^{-1} \widetilde{\Theta}_{1}^{T} \widetilde{\Theta}_{1}+\frac{1}{2} \Gamma_{2}^{-1} \widetilde{\Theta}_{2}^{T} \widetilde{\Theta}_{2}$

where

$\widetilde{\Theta}=\Theta^{*}-\widehat{\Theta}$

and $\Theta^{*}$ and $\widehat{\Theta}$ is the optimal weights vector and the estimate weights vector respectively. While $\Gamma_{1}, \Gamma_{2}>0$ are the learning rate.

$L(s, \widetilde{\Theta})$ can be upper bounded by 
$L(s, \widetilde{\Theta}) \leq \frac{1}{2} s^{2}+\frac{1}{2} \Gamma_{1}^{-1}\left\|\widetilde{\Theta}_{1}\right\|^{2}+\frac{1}{2} \Gamma_{2}^{-1}\left\|\widetilde{\Theta}_{2}\right\|^{2}$

Differentiating (16) w.r.t. time yields:

$$
\begin{aligned}
& \dot{L}(s, \widetilde{\Theta})=s \dot{s}-\Gamma_{1}^{-1} \widetilde{\Theta}_{1}^{T} \dot{\widetilde{\Theta}}_{1}-\Gamma_{2}^{-1} \widetilde{\Theta}_{2}^{T} \dot{\widetilde{\Theta}}_{2} \\
& =s \dot{S}-\Gamma_{1}^{-1} \widetilde{\Theta}_{1}^{T} \dot{\widehat{\Theta}}_{1}-\Gamma_{2}^{-1} \widetilde{\Theta}_{2}^{T} \dot{\Theta}_{2} \\
& =s\left[C A \boldsymbol{e}+C\left(A-A_{m}\right) \boldsymbol{x}_{m}+C F\right. \\
& \left.-C B_{m} \psi_{r}+C B \delta(t)\right]-\Gamma_{1}^{-1} \widetilde{\Theta}_{1}^{T} \dot{\widehat{\Theta}}_{1} \\
& -\Gamma_{2}^{-1} \widetilde{\Theta}_{2}^{T} \dot{\Theta}_{2} \\
& =-s C B K_{p} s+s C A \boldsymbol{e}+s C(A- \\
& \left.A_{m}\right) \boldsymbol{x}_{m}+s C F-s C B_{m} \psi_{r}- \\
& s C B \frac{\operatorname{sgn}(s)}{c_{n}}\left(\widehat{\Phi}_{1}\left(\boldsymbol{x} \mid \widehat{\Theta}_{1}\right)\right)^{2}[|C A \boldsymbol{e}|+ \\
& \left.\left|C\left(A-A_{r}\right) \boldsymbol{x}_{m}\right|+\left|C B_{m} \psi_{r}\right|\right]- \\
& \text { sCB sgn }(s)\left(\widehat{\Phi}_{1}\left(\boldsymbol{x} \mid \widehat{\Theta}_{1}\right) \widehat{\Phi}_{2}\left(\boldsymbol{x} \mid \widehat{\Theta}_{2}\right)\right)- \\
& \Gamma_{1}^{-1} \widetilde{\Theta}_{1}^{T} \dot{\widehat{\Theta}}_{1}-\Gamma_{2}^{-1} \widetilde{\Theta}_{2}^{T} \dot{\widehat{\Theta}}_{2}
\end{aligned}
$$

Assume that the learning laws defined as follow: $\dot{\hat{\Theta}}_{1}=\Gamma_{1} \operatorname{sgn}(s) s \zeta_{1}(\boldsymbol{x})[|C A \boldsymbol{e}|+\mid C(A-$ $\left.\left.A_{m}\right) \boldsymbol{x}_{m}|+| C B_{m} \psi_{r} \mid\right]$

$\dot{\widehat{\Theta}}_{2}=\Gamma_{2} s g n(s) s c_{n} \zeta_{2}(\boldsymbol{x})$

Then (19) can be written as below:

$$
\begin{aligned}
& \dot{L}(s, \widetilde{\Theta})=-s C B K_{p} s- \\
& \operatorname{sgn}(s) s K(\boldsymbol{x})\left[|C A \boldsymbol{e}|+\left|C\left(A-A_{m}\right) \boldsymbol{x}_{m}\right|+\right. \\
& \left.\left|C B_{m} \psi_{r}\right|\right] \times\left(\widehat{\Phi}_{1}\left(\boldsymbol{x} \mid \widehat{\Theta}_{1}\right)\right)^{2}+\operatorname{sgn}(s) s[|C A \boldsymbol{e}|+ \\
& \left.\left|C\left(A-A_{m}\right) \boldsymbol{x}_{m}\right|+\left|C B_{m} \psi_{r}\right|\right] \widehat{\Phi}_{1}\left(\boldsymbol{x} \mid \widehat{\Theta}_{1}\right)- \\
& \operatorname{sgn}(s) s c_{n}\left[K(X) \widehat{\Phi}_{1}\left(\boldsymbol{x} \mid \widehat{\Theta}_{1}\right) \widehat{\Phi}_{2}\left(\boldsymbol{x} \mid \widehat{\Theta}_{2}\right)-\right. \\
& \left.\widehat{\Phi}_{2}\left(\boldsymbol{x} \mid \widehat{\Theta}_{2}\right)\right]+\left(s C A \boldsymbol{e}+s C\left(A-A_{m}\right) \boldsymbol{x}_{m}-\right. \\
& \left.s C B_{m} \psi_{r}\right)-\operatorname{sgn}(s) s[|C A \boldsymbol{e}|+\mid C(A- \\
& \left.\left.A_{m}\right) \boldsymbol{x}_{m}|+| C B_{m} \psi_{r} \mid\right] \widehat{\Phi}_{1}\left(\boldsymbol{x} \mid \Theta_{1}^{*}\right)-\left[s c_{n} d(\boldsymbol{x})+\right. \\
& \left.\operatorname{sgn}(s) s c_{n} \widehat{\Phi}_{2}\left(\boldsymbol{x} \mid \Theta_{2}^{*}\right)\right]
\end{aligned}
$$

The first term in (22) is:

$-s C B K_{p} s$

$=-s c_{n} K(\boldsymbol{x}) K_{p} s$

While $c_{n}, K(\boldsymbol{x})$ and $K_{p}>0$ then

$-s c_{n} K(\boldsymbol{x}) K_{p} s \leq 0$

For further analysis, it is assumed that for the two continuous functions $\Phi_{1}(\boldsymbol{x})$ and $\Phi_{2}(\boldsymbol{x})$ defined in
(10) and (11) on a compact set, there exist two optimal weight vectors $\Theta_{1}^{*}$ and $\Theta_{2}^{*}$ such that:

$$
\begin{aligned}
& \left|\varepsilon_{1}(\boldsymbol{x})\right|=\left|\widehat{\Phi}_{1}\left(\boldsymbol{x} \mid \Theta_{2}^{*}\right)-\Phi_{1}(\boldsymbol{x})\right|<\delta_{1} \\
& \left|\varepsilon_{2}(\boldsymbol{x})\right|=\left|\widehat{\Phi}_{2}\left(\boldsymbol{x} \mid \Theta_{2}^{*}\right)-\Phi_{2}(\boldsymbol{x})\right|<\delta_{2}
\end{aligned}
$$

And the uncertainty bounds $K_{l}(\boldsymbol{x})$ and $d_{u}(\boldsymbol{x})$ meet the following inequalities on the compact set:

$0<K_{l}(\boldsymbol{x})<\frac{1}{1-\delta_{1}}$

$d_{u}(\boldsymbol{x})-|d(\boldsymbol{x})|>\delta_{2}$

So, (22) can be written as below:

$$
\begin{aligned}
& \dot{L}(s, \widetilde{\Theta})=-s c_{n} K(\boldsymbol{x}) K_{p} s- \\
& K_{l}(\boldsymbol{x})|s|\left[\left(\widehat{\Theta}_{1}^{T}(0)-\Theta_{1}^{* T}\right) \zeta_{1}(\boldsymbol{x})+\right. \\
& \Gamma_{1}\left(\int _ { 0 } ^ { t } \left(|C A e|+\left|C\left(A-A_{m}\right) \boldsymbol{x}_{m}\right|+\right.\right. \\
& \left.\left.\left.\left.\left|C B_{m} \psi_{r}\right|\right)|s| \zeta_{1}(\boldsymbol{x})\right) d t\right) \zeta_{1}(\boldsymbol{x})-\delta_{1}\right][|C A \boldsymbol{e}|+ \\
& \left.\left|C\left(A-A_{m}\right) \boldsymbol{x}_{m}\right|+\left|C B_{m} \psi_{r}\right|\right] \widehat{\Phi}_{1}\left(\boldsymbol{x} \mid \widehat{\Theta}_{1}\right)- \\
& |s|\left(-K(\boldsymbol{x}) \widehat{\Phi}_{1}\left(\boldsymbol{x} \mid \widehat{\Theta}_{1}\right)+1\right)|s| c_{n} \widehat{\Phi}_{2}\left(\boldsymbol{x} \mid \widehat{\Theta}_{2}\right)- \\
& |s|\left(K_{l}^{-1}(\boldsymbol{x})-\left(1+\delta_{1}\right)\right)[|C A \boldsymbol{e}|+\mid C(A- \\
& \left.\left.A_{m}\right) \boldsymbol{x}_{m}|+| C B_{m} \psi_{r} \mid\right]-|s| c_{n}\left[\varepsilon_{2}(\boldsymbol{x})+\delta_{2}\right]
\end{aligned}
$$

Its guarantee $\dot{L}(s, \widetilde{\Theta})<0$. The state errors of the system are ensured to converge to zero. This complete the proof.

\section{SIMULATION Results}

This section presents the results of a numerical simulation of the proposed combined neural network sliding mode and PD control performed to evaluate for ship steering controller and verify the stability of the system and the learning law.

The dynamics model of a warship traveling at 16 knots, considered has the following parameters $[7,10,13]$ :

$$
\begin{aligned}
K & =0.0107 \\
d_{0} & =0 \\
d_{1} & =9.42 \\
d_{2} & =0 \\
d_{3} & =2.24
\end{aligned}
$$

The reference model is designed such that,

$$
\begin{array}{ll}
k_{m} & =0.025 \\
a_{m} & =0.45 \\
b_{m} & =0.025
\end{array}
$$


The controller error metric vector $C=\left[\begin{array}{ll}10 & 1\end{array}\right]$ and $K_{p}=55$. The two RBF neural networks have fifteen neurons with the following parameters:

Initial output weight vectors $\widehat{\Theta}_{1}(0), \widehat{\Theta}_{2}(0)$ and standard deviation vector $\sigma$ are all set to 0.5 . Gaussian membership function mean values are given below:

$C_{1}=C_{2}=[i, j]^{T}$

where $i=0,1,2$ and $j=-2,-1,0,1,2$

The selected learning rate $\Gamma_{1}=\Gamma_{2}=0.1$

The initial course angle of the ship is taken as $\psi(0)=-5^{\circ}$ while other initial values of the system and the reference model are set to zero.

Fig. 3 (a) - (c) shows the simulation results for course angle tracking as a square wave input. The solid line represents the actual output; the dashed line represent the reference model output. Fig. 4 (a) - (c) shows the simulation results for course tracking as sine wave input. The solid line represents the actual output; the dashed line represent the reference model output. It can be noted that the controller output is not smooth and exhibit a ringing or chattering phenomena and this is due to the existences of the sign function in the control law.
Many methods are suggested to reduce the ringing, where a low-pass filtering of the control signal to reduce the chattering proposed in [14], while [15] introduced a sliding mode controller with uncertainty and disturbance estimator which is used to reduce the chattering. In this paper a method based on introducing a boundary layer around the switching surface is used, where a continuous control is applied within the boundary [16] [17], the control signal chattering can be removed by replacing the $\operatorname{sgn}(s)$ term in the control law by $\operatorname{sat}(s, \mu)$ where

$$
\operatorname{sat}(s, \mu)=\left\{\begin{array}{c}
\frac{s(t)}{\mu} \quad \text { if }|s(t)|<\mu \\
\operatorname{sgn}(s(t)) \quad \text { otherwise }
\end{array}\right.
$$

where $\mu$ is the boundary layer thickness which is a positive design number.

Fig. 5 (a) - (c) and Fig. 6 (a) - (c) show the simulation results after introducing the boundary layer to reduce the chattering in the controller output, and a smooth control signal has been achieved.
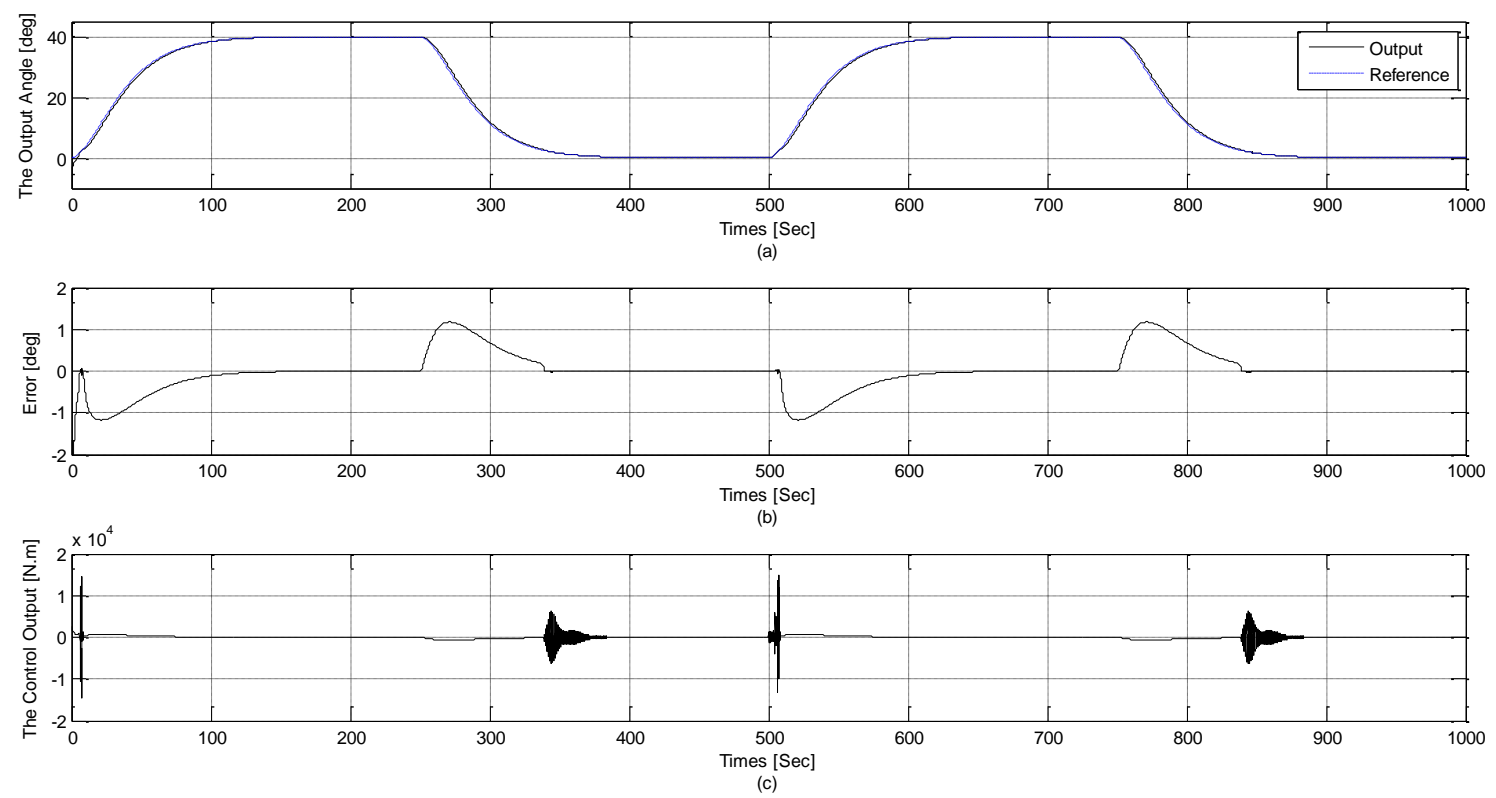

Fig. (3) (a) The ship heading angle. (b) The output tracking error. (c) The controller Output 

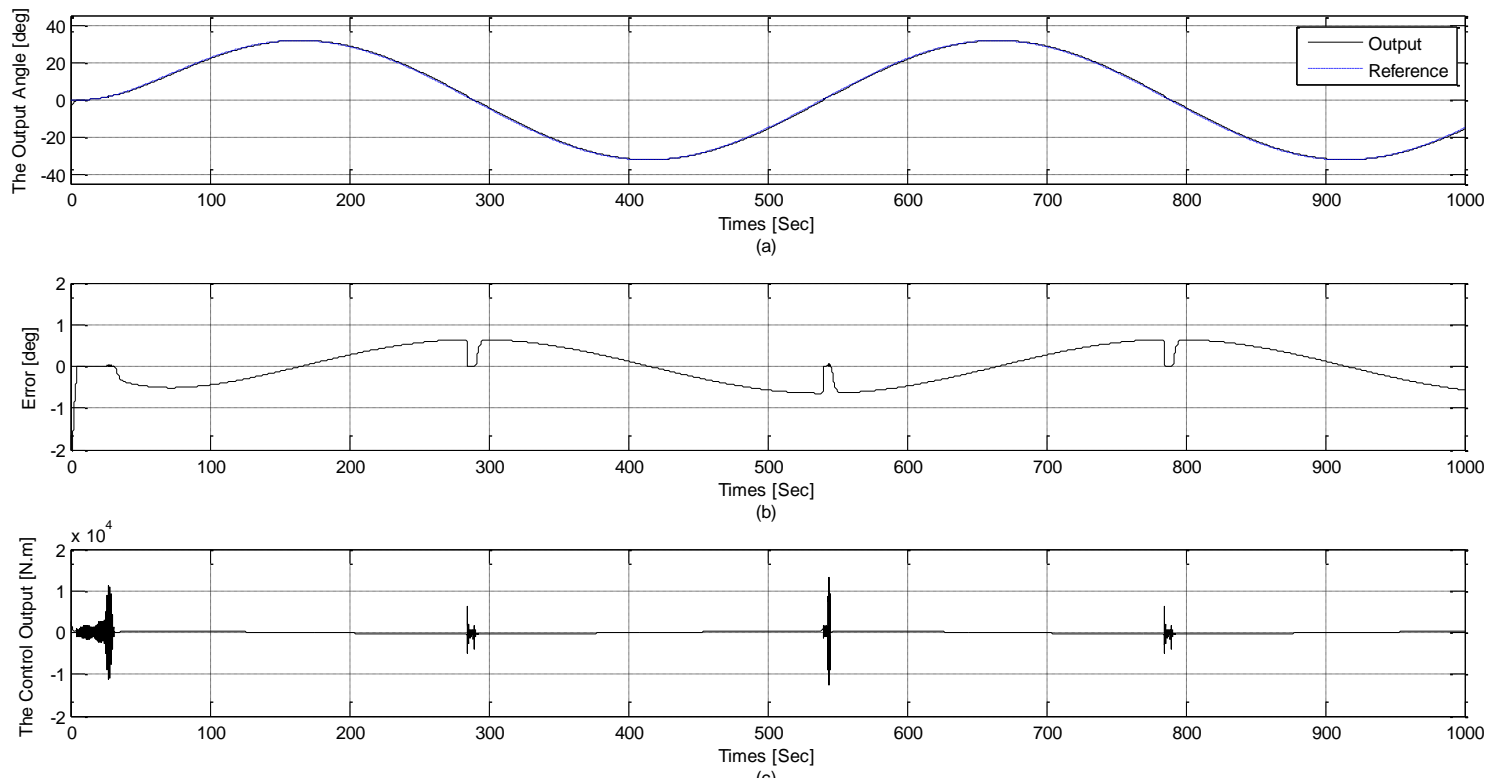

Fig. (4) (a) The ship heading Angle. (b) The output tracking error. (c) The controller Output


Fig. (5) (a) The ship heading Angle. (b) The output tracking error. (c) The controller Output 

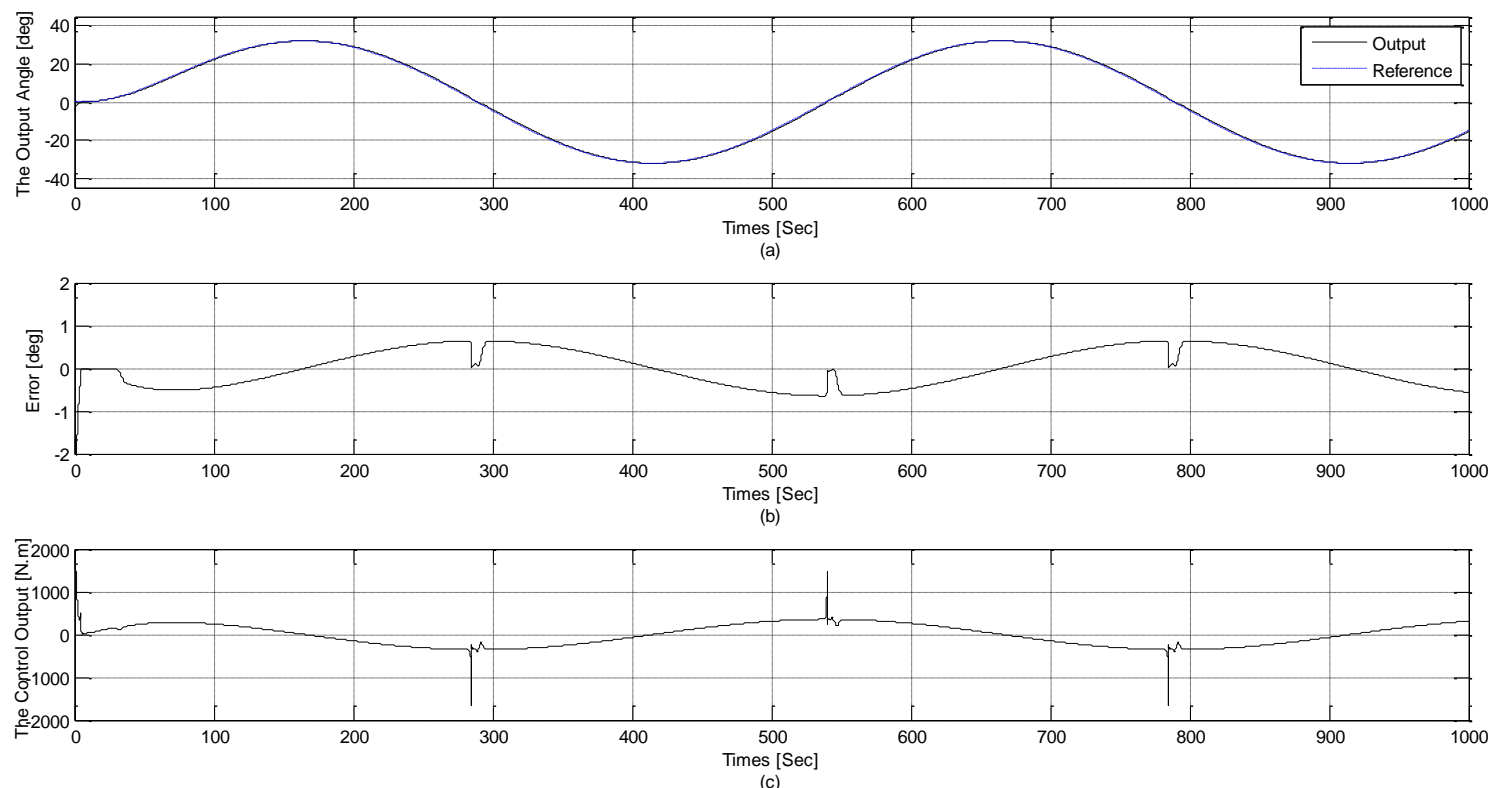

Fig. (6) (a) The ship heading Angle. (b) The output tracking error. (c) The controller Output

\section{Conclusion}

A combined RBF neural network sliding mode and PD adaptive tracking controller was proposed for controlling the directional heading course of a ship. The RBF neural networks can adaptively learn the ship uncertainty bounds, then their output used as a part of the control law, the second part is using a PD term which used to reduce the effect of the approximation error between the RBF and ship dynamics. Including Lyapunov stability theory in the design of RBF NN SMC and PD controller enables the use of the proposed control law to ensure stability and robust ship system. To reduce the ringing phenomena associated with the sliding mode control, a boundary layer technique has been used effectively. Simulation results show that proposed NN sliding mode and PD controller can solve the ship directional head course problem effectively.

\section{References}

[1] T. Arie, M. Iton, A. Senoh,"An Adaptive Steering System for a Ship", IEEE Control System Magazine, 1986.

[2] J. Ren and L. Liu, "Adaptive Neural Network Control for Ship Steering System Using Filtered
Backstepping Design", Journal of Applied Sciences 13 (10): 1691-1697, 2013.

[3] J. Van Amerongen," Adaptive Steering of Ship-A Model Reference Approach”, 1984 International Federation of Automatic Control, Vol. 20, No. 1, pp. 3-14, 1984.

[4] Lauvdal , T. and T.I. Fossen, " Robust Adaptive Ship Autopilot with Wave Filter and Integral Action", Int. J. Adaptive Control Signal Process., 12:605-622, 1998

[5] Fossen, T.I and J.P. Strand "Tutorial on Nonlinear Backstepping: Applications to Ship Control", Mod. Identif. Control, 20:83-135, 1999. [6] H. Yaozhen, X. Hairong, and Z. Fengyu,"A Fuzzy variable Structure Controller and Its Application on Ship Course Nonlinear System", 2011 Eighth International Conference on Fuzzy Systems and Knowledge Discovery (FSKD 2011).

[7] J. Cheng, J. Yi and D. Zhao," Neural Network Based Model Reference Adaptive Control for Ship Steering System", International Journal of Information Technology, Vo. 11 No. 6, 2005.

[8] Layne, J. R. and K. M. Passino, " Fuzzy Model reference Learning Control for Cargo Ship Steering", IEEE Control System Magazine, Vol. 13, No. 6, pp. 23-34, 1993. 
[9] H. Yaozhen, X. Hairong, P. Weigang, W.

Changshun and Z. Fengyu,"A Fuzzy Sliding Mode Controller and Its Application on Ship Course Control", 2010 Seventh International Conference on Fuzzy Systems and Knoledge Discovery (FSKD 2010).

[10] Le, M.D.: Online Estimation of Ship Steering Dynamics and Its Application in Designing An Optimal Autopilot", Proc. Of IFAC Computer Aided Control Sys. Design, CACSD2000, Vol. 1, pp. 7-12, 2000.

[11] La Salle, J., Lefschetz, S., "Stability By Lyapunov: Direct Method With Applications", New York,: Academic, 1961.

[12] M. Zhihong, H. R. Wu, and M. Palaniswami, "An Adaptive tracking Controller Using Neural Networks for a Class of Nonlinear Systems", IEEE Trans. On Neural Networks, Vol. 9, No. 5, September, 1998.

[13] Cheng, F., "Back-Propagation Neural Network for Nonlinear Self-Tuning Adaptive Control”, IEEE Contr. System. Mag., Vol. 10, pp. 44-48, Apr., 1990.

[14] M. L. Tseng and M. S. Chen "Chattering Reduction of Sliding Mode Control by Low-Pass Filtering The Control Signal", Asian Journal of Control, Vol. 12, No. 3, pp. 392 398, May 2010.

[15] P. V. Suryawanshi , P. D. Shendge, and S. B. Phadke, "A Boundary Layer Sliding Mode Control Design For Chatter Reduction Using Uncertainty and Disturbance Estimator", International Journal of Dynamics and Control, Volume 4, Issue 4, pp 456-465, Dec. 2016.

[16] J.-J. E. Slotine and W. Li, "Applied Nonlinear Control". Englewood Cliffs, NJ: Prentice Hall, 1991.

[17] J.-J. E. Slotine and S. S. Sastry, "Tracking Control of Nonlinear Systems Using Sliding Surfaces With Applications to Robot Manipulators,” Int. J. Contr., vol. 39, no. 2, 1983. 